
for invasive phenotype in Vegf-deleted

SUBJECT AREAS:

CANCER MODELS

MICROENVIRONMENT

ANGIOGENESIS

GENE REGULATION

Received

10 April 2012

Accepted

18 June 2012

Published

4 July 2012

Correspondence and requests for materials should be addressed to M.I. (inove-ma2@mc. pref.osaka.jp) \section{islet cell tumors}

\author{
Takaaki Takeda ${ }^{1,3}$, Hiroaki Okuyama², Yasuko Nishizawa ${ }^{2,3}$, Shuhei Tomita ${ }^{4}$ \& Masahiro Inoue ${ }^{1,3}$
}

'Department of Biochemistry, ${ }^{2}$ Pathology, Osaka Medical Center for Cancer and Cardiovascular Diseases, Higashinari-ku, Osaka 537-8511, Japan, ${ }^{3}$ Department of Clinical and Experimental Pathophysiology, Osaka University, Graduate School of Pharmaceutical Sciences, Suita, Osaka 565-0871, Japan, ${ }^{4}$ Department of Pharmacology, Institute of Health Biosciences, The University of Tokushima, Tokushima 770-8503, Japan.

In the mouse model of pancreas endocrine tumor, loss of $\operatorname{Vegf}(\mathrm{VKO})$ results in dramatically decreased tumor progression; however, the residual microscopic lesions show increased invasion into surrounding

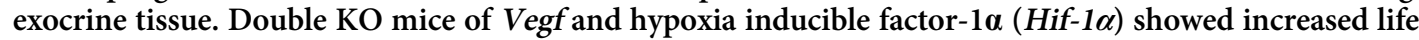
span and suppressed tumor growth due to increased apoptosis. The increased invasiveness of tumors in VKO mice was diminished in DKO mice to the levels of wild-type mice. Compared to VKO mice, DKO mice also exhibited smaller changes in the expression levels of adhesion molecules, including E-cadherin, $\mathrm{N}$-cadherin, and NCAM. These changes of adhesion molecules were not observed in the primary culture of the tumor cells under hypoxic conditions. Thus, the invasive phenotype observed under angiogenesis inhibition requires $H$ if- $1 \alpha$, but is not directly caused by acute hypoxia.

$\mathrm{V}$ EGF pathway inhibitors that impair tumor angiogenesis and growth are currently in clinical use ${ }^{1}$. Recently, we and others have reported that angiogenesis inhibition in murine tumor models leads to promotion of tumor invasion and metastasis ${ }^{2,3}$. The RIP1-Tag2 transgenic mouse model overexpresses SV40 T antigen under the control of the insulin promoter, resulting in development of islet cell tumors ${ }^{4}$. We demonstrated that VEGF-targeted drugs, as well as gene deletion of Vegf, inhibit primary tumor growth but promote tumor invasiveness and metastasis in the RIP1-Tag2 model ${ }^{2}$. VEGF inhibition generates widely hypoxic regions ${ }^{1}$ and there is a well-documented link between hypoxia and invasive phenotype ${ }^{5,6}$, thus, we speculated that hypoxia contributes to the invasive phenotype in the RIP1-Tag2 model ${ }^{1}$.

Hypoxia inducible factor- $1 \alpha$ (HIF-1 $\alpha)$ is a transcription factor that regulates gene expression under hypoxic conditions $s^{7}$. HIF- $1 \alpha$ modulates the expression of genes involved in tumorigenesis, such as angiogenesis, metabolism, apoptosis/survival, and invasion/metastasis ${ }^{7}$. HIF protein is constitutively synthesized, but degraded under normoxic conditions by the ubiquitin/proteasome system; under hypoxic conditions it is stabilized because the degradation machinery requires molecular oxygen. Once stabilized, HIF- $1 \alpha$ heterodimerizes with HIF- $1 \beta$ to act as a transcription factor, controlling a wide range of genes involved in the hypoxic response. HIF-regulated hypoxic responses are involved in cell motility, as well as invasion and metastasis in vivo ${ }^{8-10}$. Indeed, clinical studies have demonstrated a correlation between high HIF expression and poor clinical prognosis ${ }^{11-14}$.

The alterations of the microenvironment following angiogenesis inhibition are poorly understood; they likely include complex changes in oxygen tension, reactive oxygen species, metabolites, and growth factors. Many of these changes involve HIF- $1 \alpha^{7}$, thus we speculated that HIF- $1 \alpha$ might play an important role in the microenvironment where angiogenesis is disturbed. Here, using a genetic targeting strategy, we investigated the contribution of Hif- $1 \alpha$ to the changes of tumor phenotype in the Vegf-deleted islet tumors in RIP1-Tag2 mice.

\section{Results}

Hif-1 $\alpha$ deletion in the RIP1-Tag2/VKO tumors. Conditional deletion of Hif- $1 \alpha$ in insulin-secreting cells was performed using the Cre/lox system ${ }^{15,16}$. No remarkable changes were observed in the islet-specific Hif-1 $\alpha$-null mice (RipCre/Hif- $1 \alpha^{f l / f l}$ ) as previously reported ${ }^{17,18}$. Hif-1 $\alpha$ deletion in the tumors of RIP1-Tag2/RipCre/Hif-1 $\alpha^{f l / f l}$ (RIP1-Tag2/HKO) mice was confirmed at the levels of genome, mRNA, and protein (see Supplementary Fig. S1). Next, we generated RIP1-Tag2 mice lacking both Vegf and Hif-1 $\alpha$, RIP1-Tag2/RipCre/Hif-1 $\alpha^{f l f} /$ Vegf $f^{l / f l}$ (RIP1Tag2/DKO). 
a
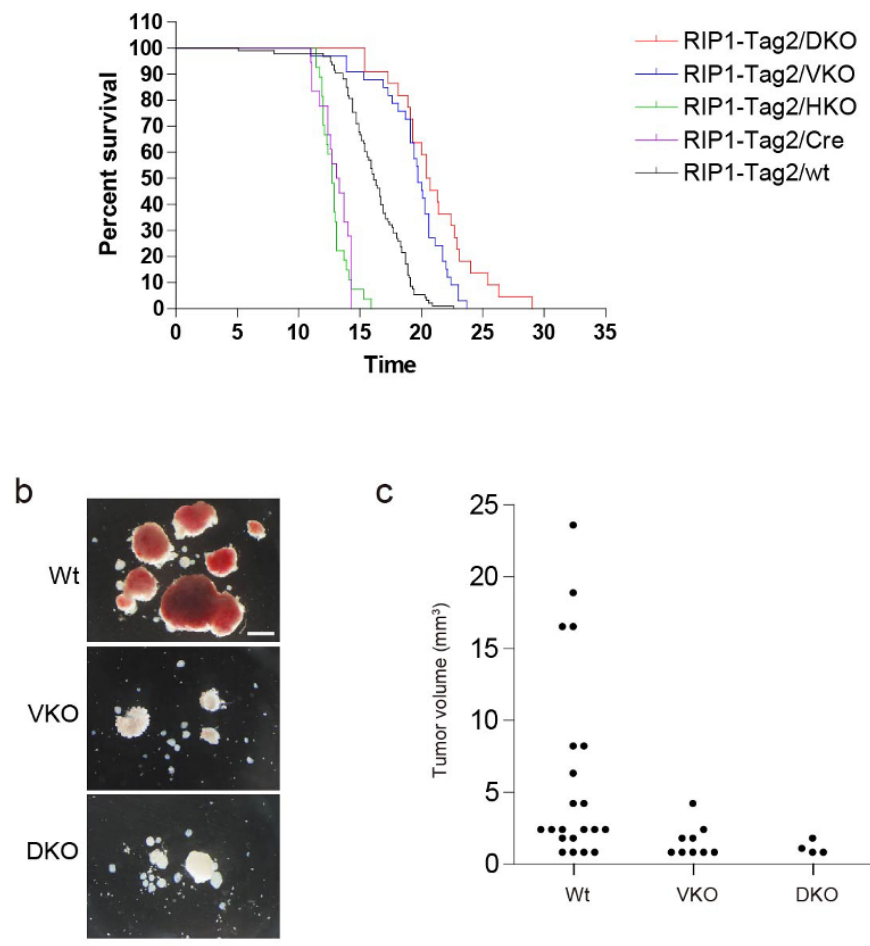

C
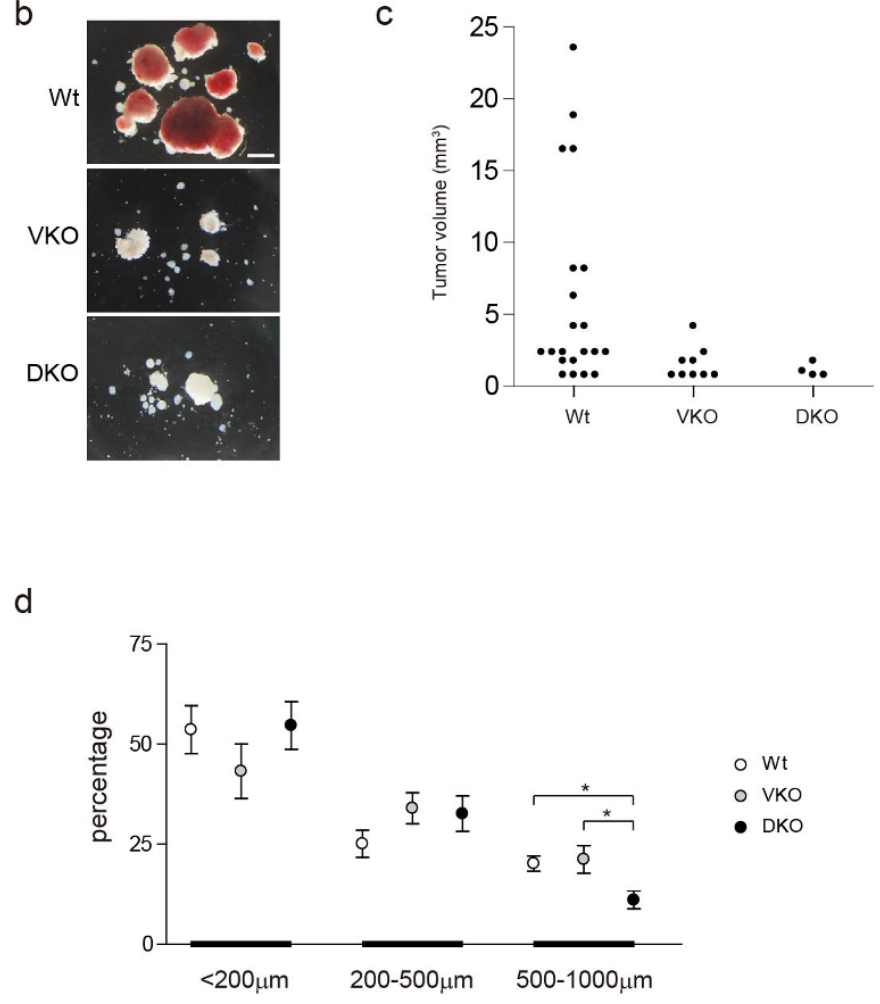

Figure $1 \mid$ Hif-1 $\alpha$ was necessary for growth of RIP1-Tag2/VKO tumors. (a) Kaplan-Meier survival curves of the indicated transgenic mice. (b) Macroscopic pictures of the isolated tumors. Scale bar, $2 \mathrm{~mm}$. (c) Volume of tumors with maximum diameters $>1 \mathrm{~mm}$. (d) Size distribution of microscopic tumors. Values are mean and $95-\%$ confidence intervals. ${ }^{*} P<0.05$.

We examined the life span of the compound transgenic mice (Fig. 1a). The life spans of the RIP1-Tag2/HKO mice ( $n=27$, median 12.7 weeks) and RIP1-Tag2/RipCre/Hif-1 $\alpha^{w t / w t}$ (RIP1-Tag2/Cre) mice ( $n=18$, median 13.2 weeks) were almost the same, but were significantly $(\mathrm{p}<0.0001)$ shorter than that of RIP1-Tag2/RipCre(-) (RIP1-Tag2/wt) mice ( $n=93$, median 16.1 weeks). RIP1-Tag2/ RipCre/Vegfllfl (RIP1-Tag2/VKO) mice ( $n=33$, median 19.7 weeks) lived significantly $(\mathrm{p}<0.0001)$ longer than the RIP1-Tag2/wt mice. RIP1-Tag2/DKO mice ( $n=22$, median 20.6 weeks) lived slightly but significantly $(\mathrm{p}<0.05)$ longer than RIP1-Tag2/VKO mice.

Microscopic analysis revealed that the tumor progression was equally accelerated in RIP1-Tag2/Cre and RIP1-Tag2/HKO mice, although the morphology of these tumors at each stage was the same as that of the RIP1-Tag2/wt tumors (see Supplementary Fig. S2). These results indicate that forced expression of Cre recombinase in the insulin-secreting cells accelerated tumor progression in RIP1Tag2 mice, and deletion of Hif- $1 \alpha$ did not have a remarkable effect on RIP1-Tag2/Cre tumors.

At 13-15 weeks, the invasive phenotype is remarkably increased in RIP1-Tag2/VKO mice ${ }^{4}$. As most of the RIP1-Tag2/Cre and
RIP1-Tag2/HKO mice died at this time point, we compared RIP1Tag2/wt, RIP1-Tag2/VKO, and RIP1-Tag2/DKO at 13-15 weeks for further analysis of the invasive phenotype.

Tumor growth was suppressed in the RIP1-Tag2/DKO tumors. Firstly, the tumors were macroscopically examined. The RIP1-Tag2/ wt tumors were red in color with a smooth surface, while the tumors in RIP1-Tag2/VKO mice were white in color with a rough surface (Fig. 1b). Tumors greater than $2 \mathrm{~mm}^{3}$ in volume were rarely observed in RIP1-Tag2/VKO mice (Fig. 1c). In RIP1-Tag2/DKO mice, the tumors were whitish, as in RIP1-Tag2/VKO mice, and all of the tumors were less than $2 \mathrm{~mm}^{3}$ in volume. Next, the tumors were microscopically examined. Size distribution of microscopic tumors was about the same between RIP1-Tag2/wt and RIP1-Tag2/VKO mice (Fig. 1d), in contrast to the drastically decreased number of larger macroscopic tumors in RIP1-Tag2/VKO (Fig. 1c). These results indicate that tumors can grow at microscopic levels despite decreased microvessels ${ }^{16}$. Meanwhile, ratio of larger microscopic lesions in RIP1-Tag2/DKO mice was significantly less than RIP1Tag2/VKO mice (Fig. 1d). 
a
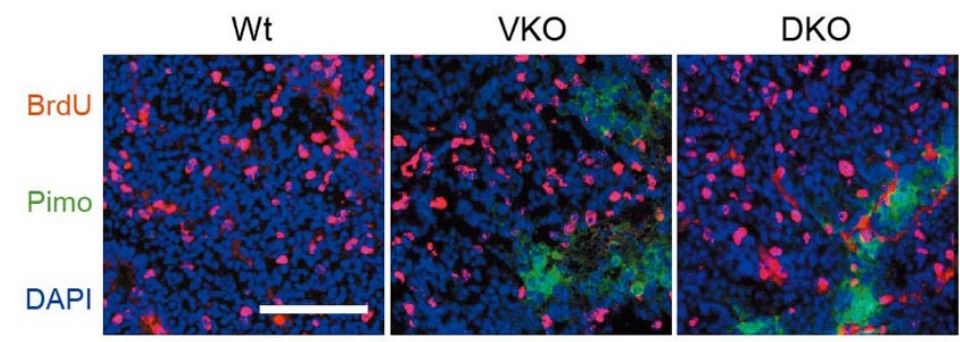

b
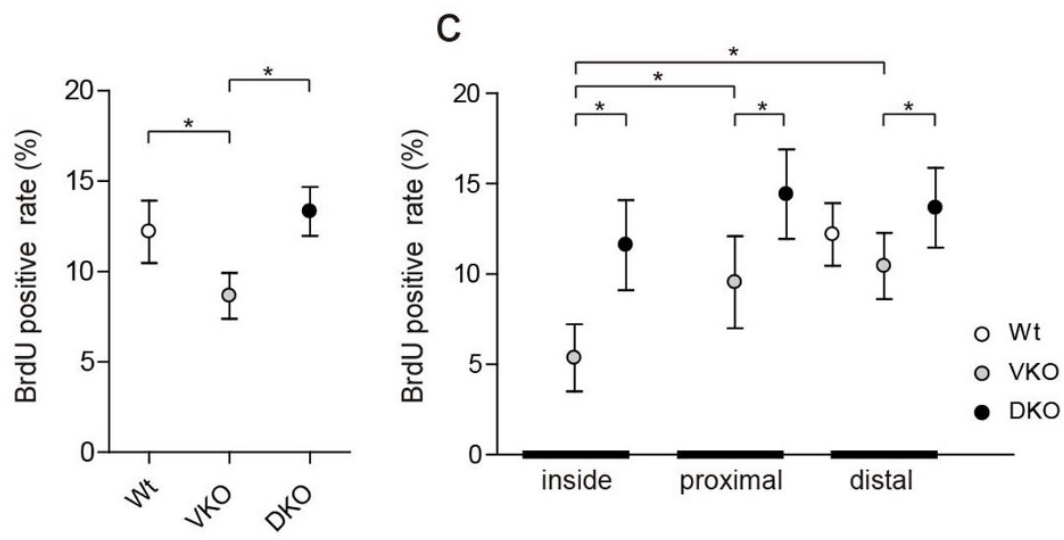

d
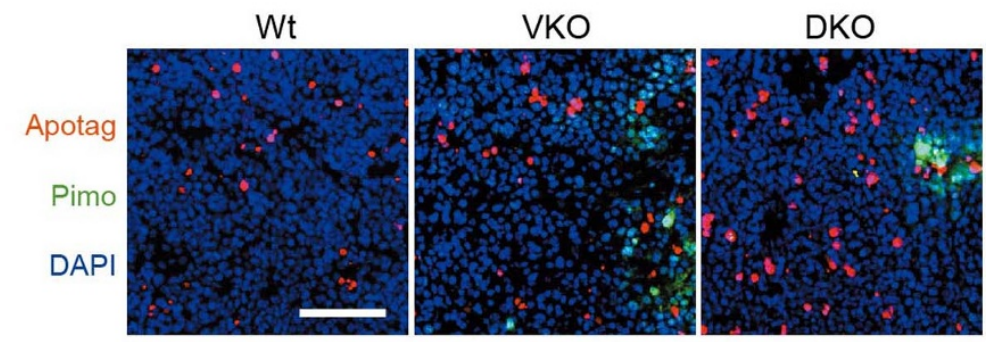

e

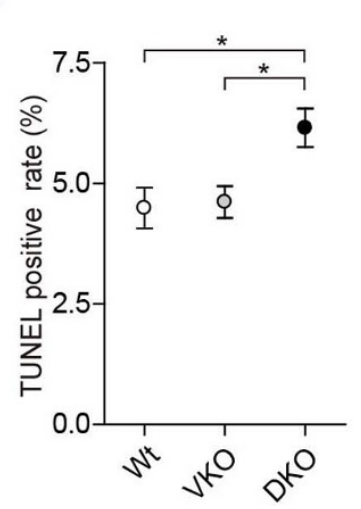

f

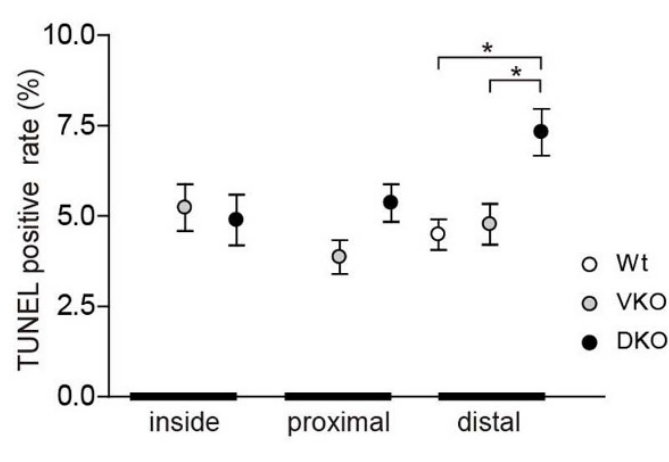

Figure $2 \mid$ Hif-1 $\alpha$ was necessary for survival of cancer cells in RIP1-Tag2/VKO tumors. (a) Immunohistochemistry of the tumors. Red, BrdU; green, pimonidazole; blue, DAPI. Scale bar, $100 \mu \mathrm{m}$. (b) Quantification of BrdU uptake in the overall RIP1-Tag2 lesions. Values are mean and 95-\% confidence intervals. (c) Quantification of BrdU incorporation in different locations. Values are mean and 95-\% confidence intervals. (d) TUNEL staining of RIP1Tag2 tumors. Red, Apoptag; green, pimonidazole; blue, DAPI. Scale bar, $100 \mu \mathrm{m}$. (e) Quantification of cell death in the overall RIP1-Tag2 lesions. Values are mean and 95-\% confidence intervals. (f) Quantification of cell death in locations relative to the pimonidazole-positive areas, as in (d). Values are mean and $95-\%$ confidence intervals. Statistical analaysis was performed between the pooled areas from different genotypes.

Hif-1 $\alpha$ was necessary for survival of RIP1-Tag2/VKO tumor cells. We investigated the mechanism underlying the reduced number of larger microscopic lesions in RIP1-Tag2/DKO mice. Microvessel area was dramatically decreased in the tumors of RIP1-Tag2/VKO compared with RIP1-Tag2/ $\mathrm{wt}^{16}$, but was about the same in the tumors of RIP1-Tag2/DKO and RIP1-Tag2/VKO (see Supplementary
Fig. S3), indicating that alterations in angiogenesis are not likely to be the major cause of growth suppression.

Tumor growth is determined by the balance between cell proliferation and cell death ${ }^{19}$. The overall proliferation rate in RIP1-Tag2/ VKO mice was significantly lower than in RIP1-Tag2/wt and RIP1Tag2/DKO mice (Fig. 2a,b). Next, we evaluated the differences of cell 
proliferation in the regions with different pimonidazole staining patterns (see Supplementary Fig. S4). The RIP1-Tag2/wt lesions were entirely pimonidazole negative, so all the regions were classified as distal. In the RIP1-Tag2/VKO lesions, the proliferation rate was decreased inside the pimonidazole positive area compared with in the proximal and distal areas (Fig. 2c). In RIP1-Tag2/DKO, the proliferation rate was higher in all lesions than in RIP1-Tag2/VKO lesions. Thus, Hif- $1 \alpha$ may suppress cell proliferation in hypoxic regions, although this does not explain the decrease of microscopically large lesions in RIP1-Tag2/DKO mice.

Next, we evaluated cell death by TUNEL staining (Fig. 2d). The overall apoptotic rate in the tumors from RIP1-Tag2/DKO mice was higher than from RIP1-Tag2/wt and RIP1-Tag2/VKO mice (Fig. 2e). When the localized differences in cell death were evaluated, the apoptotic rate of RIP1-Tag2/DKO was significantly higher in areas distant from the pimonidazole-positive areas (Fig. 2f). The same pattern was obtained with cleaved caspase- 3 staining, indicating the death is caspase-3 dependent apoptosis (Supplementary Fig. $\mathrm{S} 4 \mathrm{~b})$.These results indicate that the decrease of microscopic lesions in RIP1-Tag2/DKO mice was attributed to cell death in the relatively oxygenized regions, and that Hif- $1 \alpha$ was necessary for survival of cancer cells in these regions.

Hif-1 $\alpha$ was necessary for invasive phenotype of RIP1-Tag2/VKO tumors. To evaluate the invasive phenotype, the lesions were classified according to the previously reported grading system ${ }^{20}$ (Fig. 3a). Neither angiogenic islets nor islet tumors were observed in RIP1-Tag2/VKO and RIP1-Tag2/DKO. An increased number of invasive lesions was observed in RIP1-Tag2/VKO compared with RIP1-Tag2/wt (Fig. 3b) ${ }^{2}$, while the number of invasive legions observed in RIP1-Tag2/DKO was similar to that in RIP1-Tag2/wt. Thus, Hif- $1 \alpha$ was necessary for the invasive phenotype in the RIP1Tag2/VKO tumors.

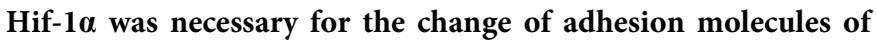
RIP1-Tag2/VKO tumors. To further investigate the mechanism underlying the invasive phenotype in RIP1-Tag2/VKO mice, we examined the expressions of adhesion molecules, including Ecadherin, N-cadherin, and NCAM. Altered adhesion molecule expressions are reportedly involved in invasiveness of cancer cells $^{21,22}$ and, moreover, involvement of E-cadherin and NCAM in the invasion and metastasis in RIP1-Tag2 tumors was previously demonstrated $^{23-25}$. In most of the RIP1-Tag2 tumors, homogenous E-cadherin, N-cadherin, and NCAM staining was observed with membrane localization ${ }^{26}$ (Fig. 3c,d). In contrast, regions of remarkably low-intensity staining of these adhesion molecules were frequently observed in RIP1-Tag2/VKO tumors (Fig. 3c,d,e). In these regions, the expressions of E-cadherin/ $\mathrm{N}$-cadherin as well as of E-cadherin/NCAM were reciprocal (Fig. 3c,d). Compared to RIP1-Tag2/VKO mice, RIP1-Tag2/DKO mice exhibited a significantly lower incidence of tumors with impaired E-cadherin expression, with levels similar to those of RIP1-Tag2/wt mice (Fig. 3c,d,e). Pimonidazole-positive staining was observed mainly in the core region of the RIP1-Tag2/VKO tumors, separate from the invasive front, and the adhesion molecule expressions were not consistently co-localized with the hypoxic regions (Fig. 3f).

Changes of the adhesion molecules in RIP1-Tag2/VKO tumors are not due to acute hypoxic response. To investigate whether the altered expressions of the adhesion molecules were due to the hypoxic response, we applied a recently developed primary culture technology, the CTOS (cancer tissue-originated spheroid) method ${ }^{27}$. CTOSs were prepared from RIP1-Tag2/wt tumors (Fig. 4a, see Supplementary Fig. S5a). The cells in CTOS shared similar morphology with the original tumor cells, and the cells stained positive for insulin (Supplementary Fig. S5b). The expression patterns of the genes associated with RIP1-Tag2 tumor progression, such as increased IGF2 ${ }^{28}$ and decreased GLUT2 ${ }^{29}$, were similar between the CTOSs and the original tumors (Supplementary Fig. S5c). Thus, CTOS preserved the characteristics of the original tumors. The cell death in the CTOSs after $24 \mathrm{~h}$ of culture was minimum and equivalent under normoxic and hypoxic conditions (Supplementary Fig. S5d). When the CTOSs were cultured in hypoxia for $24 \mathrm{~h}$, Hif- $1 \alpha$ protein levels were dramatically increased (Fig. $4 b$ ), as were expressions of Hif- $1 \alpha$ target genes, including Glut1, LdhA, and Pdk1 (Fig. 4c). In contrast, mRNA levels of E-cadherin and N-cadherin did not change after $24 \mathrm{~h}$ culture under hypoxic conditions (Fig. 4d). NCAM expression was increased in hypoxia. Whole-mount staining of CTOSs revealed that the levels and the localization of E-cadherin and N-cadherin did not change within $24 \mathrm{~h}$ (Fig. 4e). These findings, taken together with the lack of correlation between hypoxic regions and localization of changed expressions of adhesion molecules in vivo (Fig. 3f), indicate that the changed expressions of adhesion molecules was not likely to be an acute hypoxic response. Meanwhile, expressions of twist and vimentin were increased in the hypoxic CTOSs, suggesting that epithelial-mescenchymal transition (EMT) might play a role. It is also possible that exposure to hypoxic conditions for $24 \mathrm{~h}$ might not be enough to observe downregulation of E-cadherin, as the decrease of E-cadherin expression is often delayed in $\mathrm{EMT}^{30}$.

\section{Discussion}

We showed that Hif- $1 \alpha$ is necessary for the invasive phenotype of RIP1-Tag2/VKO tumors, although the mechanism of Hif- $1 \alpha$ activation is still unclear. We did not find direct evidence that Hif- $1 \alpha$ is induced by hypoxia. Conversely, pimonidazole staining was usually observed deep inside the tumors, away from the invasive front. As pimonidazole detects areas of extremely low oxygen tension, Hif- $1 \alpha$ might be activated in the pimonidazole-negative and moderately hypoxic areas, as previously reported ${ }^{31}$. It is also possible that Hif$1 \alpha$ was activated by other factors than hypoxia. Some of the growth factors are known to increase the synthesis of Hif- $1 \alpha$ protein $^{32}$. In the RIP1-Tag2/VKO tumors, impaired circulation system might result in retention of secreted molecules such as growth factors. Alternatively, Hif- $1 \alpha$ might be activated by reactive oxygen species (ROS). At the invasive front, oxygen tension should be relatively high because tumor cells are close to the normal vessels in adjacent exocrine tissue. Oxidative phosphorylation in insulin-secreting cells ${ }^{33}$ might generate ROS, which can stabilize Hif- $1 \alpha$ protein.

It also remains to be determined what downstream mechanism of Hif- $1 \alpha$ induces the invasive phenotype. Several Hif- $1 \alpha$ target genes are reportedly involved in the invasiveness of cancer cells ${ }^{34-36}$, including HGF and c-Met ${ }^{5}$. Our results indicate that it is unlikely that a direct and/or acute Hif- $1 \alpha$ response caused the invasive phenotype. One candidate mechanism for a chronic or indirect effect of Hif- $1 \alpha$ is $\mathrm{EMT}^{30}$, particularly since we observed increased expressions of twist and vimentin in primary cultured tumor cell spheroids. We did not observe decrease of E-cadherin expression after $24 \mathrm{~h}$ culture of the tumor spheroids under hypoxic conditions. Methods for long time culture are still under development. As the change of E-cadherin expression takes time in some cases compared with the transcription factors $^{30}$, it remains possible that downregulation of E-cadherin in vivo is due to EMT. In some situations, a "cadherin switch" occurs, where downregulation of E-cadherin is accompanied with upregulation of $\mathrm{N}$-cadherin ${ }^{37}$. The change in cadherin profile observed in this study might be due to trans-differentiation from beta-cells to other type of cells, which might have invasive potential. Alternatively, invasive clones might be selected by hypoxia, as the invasive tumors were observed also in RIP1-Tag2/wt tumors at low frequency. More frequently observed apoptosis in the absence of Hif- $1 \alpha$, could also suggest that HIF-1 $\alpha$ supports the survival of tumor cells in the deteriorated microenvironment due to impaired angiogenesis.

Paradoxical expression of HIF- $1 \alpha$ and VEGF is reported in invasive pancreatic endocrine tumors ${ }^{38}$. We observed that VEGF 
a

Wt

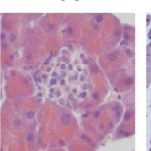

VKO

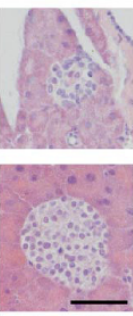

Bar 50 $\mu \mathrm{m}$

C<smiles>FC#[Te]</smiles>

E-cad

\author{
Wt
}

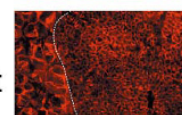

$\mathrm{H}$
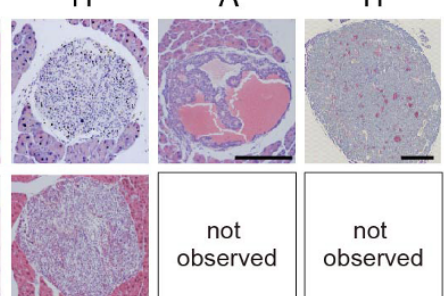

not
observed

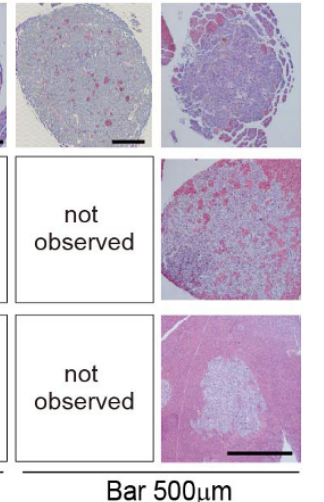

Bar 200 $\mu \mathrm{m}$

$\mathrm{N}-\mathrm{ca}$

E-cad N-cad

Hoechst

\section{VKO}
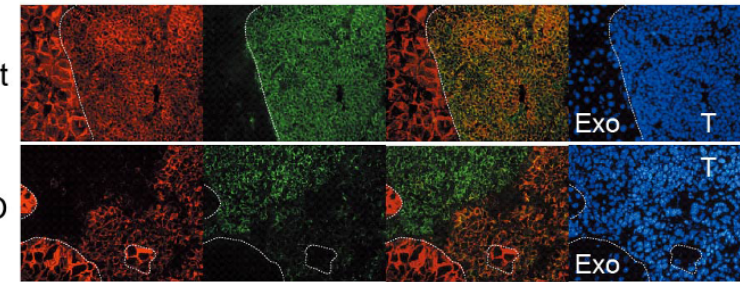

DKO

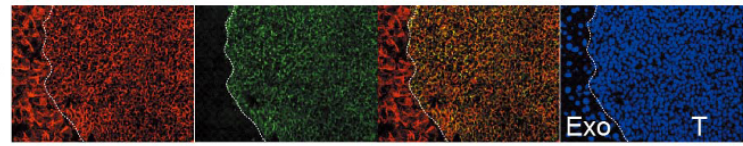

d

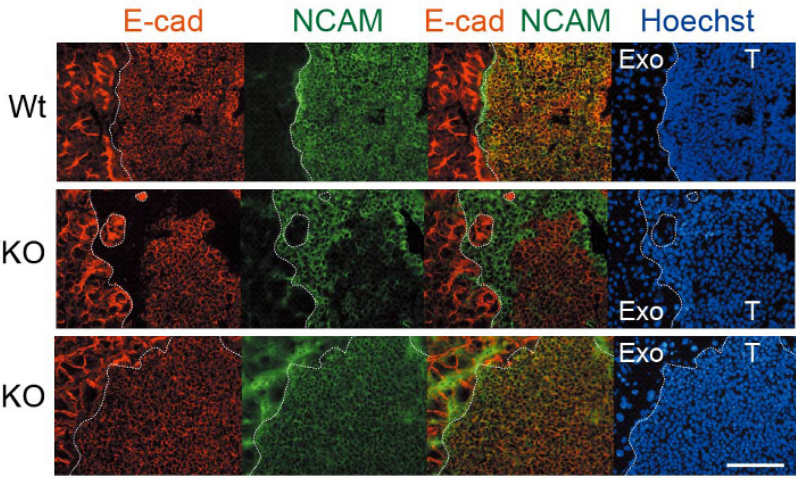

IC

$T$

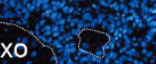

T

f

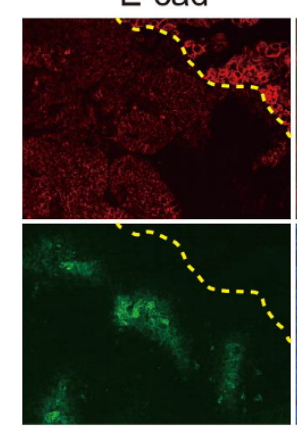

Pimo
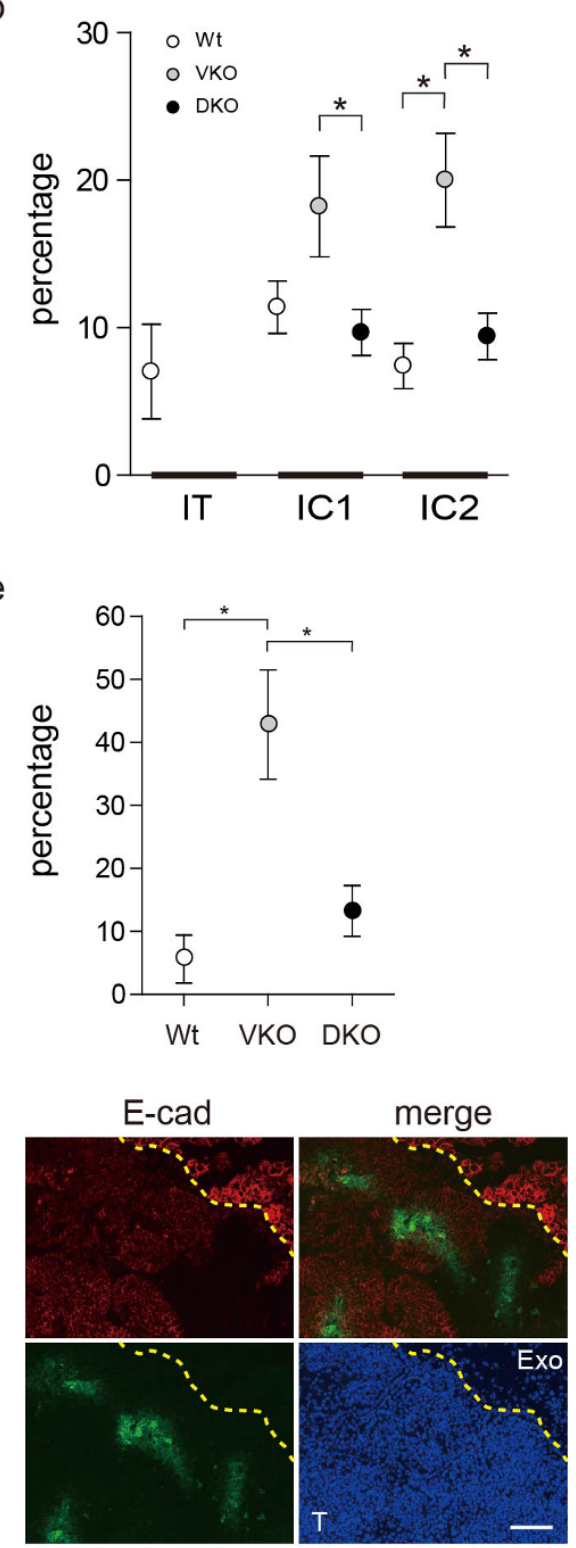

Hoechst

Figure 3 | HIF-1 $\alpha$ was necessary for invasive phenotype of RIP1-Tag2/VKO tumors. (a) Microscopic features of the tumors. Representative H\&E-stained tumors in each grade are shown. N, normal shaped lesion; H, hyperplasic; A, angiogenic; IT, insulin tumor; IC, invasive carcinoma. (b) Grading of microscopic lesions by the extent of invasion. IT, not invasive; IC1, moderately invasive; IC2, highly invasive. Values are mean and 95-\% confidence intervals. ${ }^{*} P<0.05$. (c, d) Double staining of E-cadherin and N-cadherin (c) or NCAM (d) in RIP1-Tag2/DKO tumors. Red, E-cadherin (E-cad); green, N-cadherin (N-cad) or NCAM; blue, Hoechst 33342. T, tumor; Exo, exocrine tissue. The boundary of the tumor and the exocrine pancreas is traced with a dotted line. Scale bar, $100 \mu \mathrm{m}$. (e) Frequency of tumors with mixed E-cadherin expression among the invasive tumors, shown as the percentage. Values are mean and 95$\%$ confidence intervals. (f) Immunohistochemistry of a RIP1-Tag2/VKO tumor. Red, E-cad; green, pimonidazole; blue, Hoechst33342. Scale bar, $100 \mu$ m.

expression levels did not change in HKO Rip1-Tag2 tumor (supplementary figure S6). Although islets are highly vascularized organ and it depends on the expression of $\mathrm{VEGF}^{16}$, the role of HIF in the expression of VEGF in pancreas islets might be minimum at the constitutive levels at least.

In this study, we revealed that Hif- $1 \alpha$ was necessary for the growth as well as the induction of invasive phenotype in the RIP1-Tag2/ Vegf-null tumors. Combining the antiangiogenic drugs with an HIF inhibitor might be helpful to counteract the switch to more aggressive behavior that is elicited by VEGF inhibition.

\section{Methods}

Animal study. The animal experiments were approved by the institutional committee of animal care of the Osaka Medical Center for Cancer and Cardiovascular
Diseases. Gene deletion specifically in the insulin-producing cells was achieved with the Cre/lox system ${ }^{16}$. RIP1-Tag2, Hif-1 $\alpha$ lox mice ${ }^{15}$, RipCre mice, and Vegflox mice ${ }^{16}$ were previously described. All mouse strains were back-crossed to $\mathrm{C} 57 \mathrm{Bl} / 6$ for at least 5 generations before making compound transgenic mice.

Cell culture. Cells were cultured in a humidified atmosphere of $5 \% \mathrm{CO}_{2} / 95 \%$ air at $37^{\circ} \mathrm{C}$, unless otherwise noted. Hypoxic conditions were achieved using a hypoxic workbench (INVIVO2 400, Ruskinn Technology, Bridgend, UK) to incubate cells in a humidified atmosphere of $1 \% \mathrm{O}_{2}$ and $5 \% \mathrm{CO}_{2}$ at $37^{\circ} \mathrm{C}$.

CTOS preparation and culture. Cancer tissue-originated spheroids (CTOSs) were prepared as previously described ${ }^{27}$. Briefly, tumors were dissected from pancreases, and placed in StemPro hESC SFM Complete medium (Life Technologies) on a noncoated dish. The tumors were torn with needles and the tumor fragments were cultured overnight in the medium. CTOS formation was confirmed and the spheroids were picked up with a pipet under a microscope. 
a

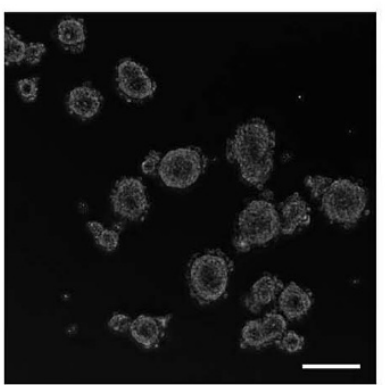

c

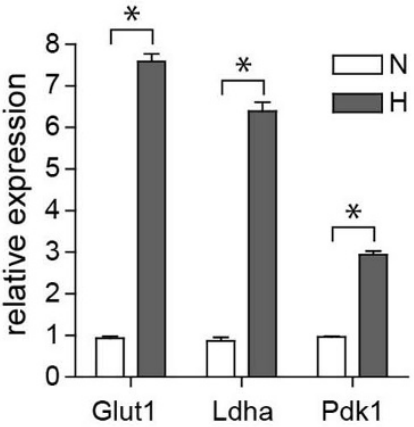

b

\section{mouse\#1 mouse\#2 mouse\#3}

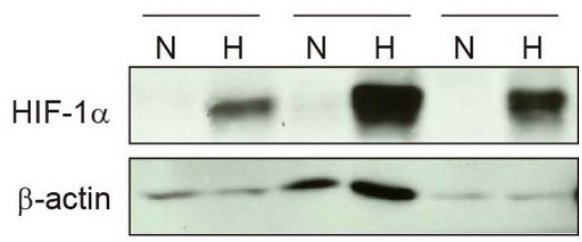

d

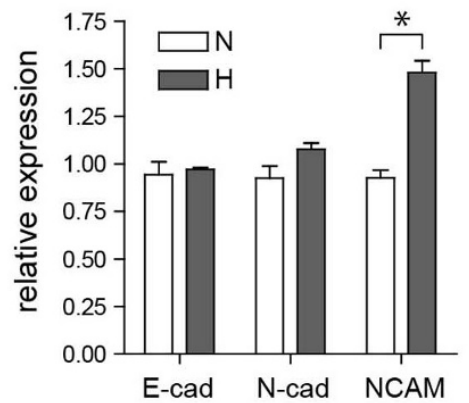

e

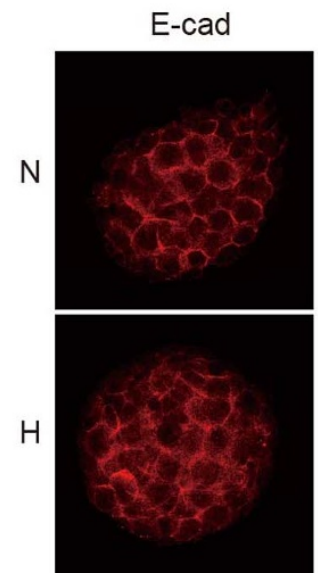

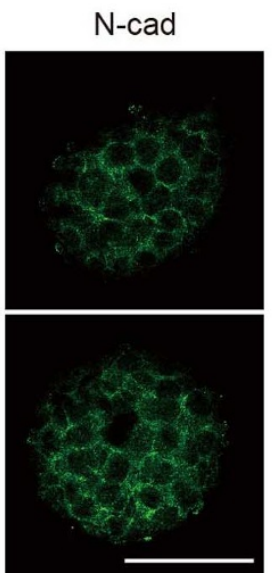

f

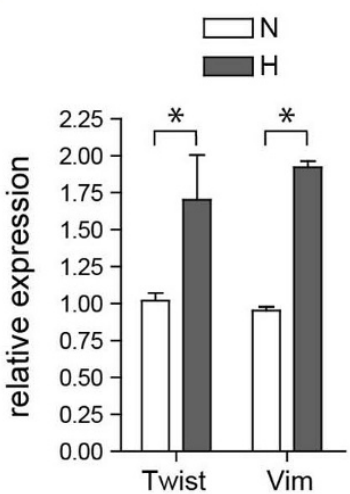

Figure $4 \mid$ Changes of the expression of the adhesion molecules were not due to acute hypoxic response. (a) Phase contrast image of CTOSs from RIP1Tag2/wt tumors. Scale bar, $100 \mu \mathrm{m}$. (b) Western blotting of HIF- $1 \alpha$ in the CTOSs cultured for $24 \mathrm{~h}$ under normoxic $(\mathrm{N})$ or hypoxic $(\mathrm{H})$ conditions. The CTOSs were pooled from the tumors of each mouse. (c, d, f) Relative levels of mRNA expression of indicated genes. E-cad, E-cadherin; N-cad, Ncadherin; Vim, vimentin. (e) Whole-mount staining of E-cadherin and N-cadherin of CTOSs. Scale bar, $50 \mu \mathrm{m}$.

RT-PCR. Total RNA was extracted using RNeasy (QIAGEN) and was reverse transcribed with SuperScript III (Invitrogen) using the pd(N)6 primer. Real-time PCR was performed using SYBR Premix ExTaq (Perfect Real time) (RR041A, TaKaRa BIO, Inc.) and the ABI PRISM 7500 Real-Time PCR System (Applied Biosystems). The sequences of the primers are provided in the supplementary information.

Western blotting. Cells were collected in ice-cold PBS, and immediately centrifuged for $5 \mathrm{~min}$ at $4^{\circ} \mathrm{C}$. The pellets were dissolved in lysis buffer containing the protease inhibitors aporotinin and leupeptin. The primary antibody was anti-HIF (NB100$105,1: 1000$, NOVUS BIOLOGICALS) and the horseradish peroxidase-conjugated secondary antibody was anti-mouse IgG (sc-2005, $1: 2500$, Santa Cruz Biotechnology, Inc.).

Tumor grading. The paraffin-embedded samples were serially sectioned into $5-\mu \mathrm{m}-$ thick slices; four sections from $50-\mu \mathrm{m}$ intervals spanning $200 \mu \mathrm{m}$ were H\&E stained. At least five mice per genotype were analyzed. Grading of the RIP1-Tag2 tumors was performed as previously described ${ }^{20}$. The well-vascularized lesions were observed, approximately $10 \%$, both in RIP1-Tag2/VKO and RIP1-Tag2/DKO mice. As we previously showed ${ }^{16}$, the lesions are 'leaky lesions', in which Vegf gene is not deleted due to incompleteness of the Cre/lox system. We removed the leaky lesions from the analysis in this study.

Immunohistochemical analysis. Mice were injected i.p. with $750 \mathrm{mg} / \mathrm{kg}$ BrdU (Roche) or $60 \mathrm{mg} / \mathrm{kg}$ Hypoxyprobe (Natural Pharmacia International Inc.), for BrdU and pimonidazole labeling experiments, respectively. After two hours, mice were sacrificed and pancreases harvested. Pancreases were embedded in OCT and frozen. The frozen samples were serially sectioned into $10-\mu \mathrm{m}$-thick slices, and subjected to each immunohistochemical analysis. The details are provided in the supplementary information.

Cell death and proliferation assay. The sections were stained with ApopTag or BrdU with DAPI and pimonidazole. Tumors were classified into three regions according to the relative location to the pimonidazole-positive area: inside, proximal, and distal (see Supplementary Fig. S4). Five square-shaped, $100-\mu \mathrm{m}^{2}$ areas were randomly selected within each region. The ratio of TUNEL-positive or BrdU-positive nuclei to total nuclei was calculated within each area. Three tumors each from at least three mice were analyzed.

Statistical analysis. Statistical analysis was performed using GraphPad Prism 4 (GraphPad Software). The values presented in graphs are mean and 95-\% confidence intervals, or mean \pm SD. The differences between groups were examined for significance with the Student's t-test, with $P$-values $<0.05$ considered to be significant. Overall survival was estimated by Kaplan-Meier methods, and examined by Logrank test.

1. Ferrara, N., Mass, R. D., Campa, C. \& Kim, R. Targeting VEGF-A to treat cancer and age-related macular degeneration. Annu Rev Med 58, 491-504 (2007). 
2. Paez-Ribes, M. et al. Antiangiogenic therapy elicits malignant progression of tumors to increased local invasion and distant metastasis. Cancer cell 15, 220-231 (2009).

3. Ebos, J. M. et al. Accelerated metastasis after short-term treatment with a potent inhibitor of tumor angiogenesis. Cancer cell 15, 232-239 (2009).

4. Hanahan, D. Heritable formation of pancreatic beta-cell tumours in transgenic mice expressing recombinant insulin/simian virus 40 oncogenes. Nature 315, 115-122 (1985).

5. Pennacchietti, S. et al. Hypoxia promotes invasive growth by transcriptional activation of the met protooncogene. Cancer cell 3, 347-361 (2003).

6. Christofori, G. New signals from the invasive front. Nature 441, 444-450 (2006)

7. Semenza, G. L. Targeting HIF-1 for cancer therapy. Nature reviews 3, 721-732 (2003).

8. Mendez, O. et al. Knock down of HIF-1alpha in glioma cells reduces migration in vitro and invasion in vivo and impairs their ability to form tumor spheres. Molecular cancer 9, 133 (2010)

9. Staller, P. et al. Chemokine receptor CXCR4 downregulated by von HippelLindau tumour suppressor pVHL. Nature 425, 307-311 (2003).

10. Muller, A. et al. Involvement of chemokine receptors in breast cancer metastasis. Nature 410, 50-56 (2001).

11. Generali, D. et al. Hypoxia-inducible factor-1alpha expression predicts a poor response to primary chemoendocrine therapy and disease-free survival in primary human breast cancer. Clin Cancer Res 12, 4562-4568 (2006).

12. Tan, E. Y. et al. Cytoplasmic location of factor-inhibiting hypoxia-inducible factor is associated with an enhanced hypoxic response and a shorter survival in invasive breast cancer. Breast Cancer Res 9, R89 (2007).

13. Rasheed, S. et al. Hypoxia-inducible factor-1alpha and -2alpha are expressed in most rectal cancers but only hypoxia-inducible factor-1alpha is associated with prognosis. British journal of cancer 100, 1666-1673 (2009).

14. Baba, Y. et al. HIF1A overexpression is associated with poor prognosis in a cohort of 731 colorectal cancers. The American journal of pathology 176, 2292-2301 (2010).

15. Tomita, S. et al. Defective brain development in mice lacking the Hif-1alpha gene in neural cells. Molecular and cellular biology 23, 6739-6749 (2003).

16. Inoue, M., Hager, J. H., Ferrara, N., Gerber, H. P. \& Hanahan, D. VEGF-A has a critical, nonredundant role in angiogenic switching and pancreatic beta cell carcinogenesis. Cancer cell 1, 193-202 (2002).

17. Zehetner, J.et al. PVHL is a regulator of glucose metabolism and insulin secretion in pancreatic beta cells. Genes \& development 22, 3135-3146 (2008).

18. Cantley, J. et al. Deletion of the von Hippel-Lindau gene in pancreatic beta cells impairs glucose homeostasis in mice. The Journal of clinical investigation 119, 125-135 (2009).

19. Naik, P., Karrim, J. \& Hanahan, D. The rise and fall of apoptosis during multistage tumorigenesis: down-modulation contributes to tumor progression from angiogenic progenitors. Genes \& development 10, 2105-2116 (1996).

20. Lopez, T. \& Hanahan, D. Elevated levels of IGF-1 receptor convey invasive and metastatic capability in a mouse model of pancreatic islet tumorigenesis. Cancer cell 1, 339-353 (2002).

21. Tomita, K. et al. Cadherin switching in human prostate cancer progression. Cancer research 60, 3650-3654 (2000).

22. McLaughlin, R. B., Jr. et al. Nerve cell adhesion molecule expression in squamous cell carcinoma of the head and neck: a predictor of propensity toward perineural spread. The Laryngoscope 109, 821-826 (1999).

23. Perl, A. K., Wilgenbus, P., Dahl, U., Semb, H. \& Christofori, G. A causal role for Ecadherin in the transition from adenoma to carcinoma. Nature 392, 190-193 (1998).

24. Du, Y. C., Lewis, B. C., Hanahan, D. \& Varmus, H. Assessing tumor progression factors by somatic gene transfer into a mouse model: $\mathrm{Bcl}-\mathrm{xL}$ promotes islet tumor cell invasion. PLoS biology 5, e276 (2007).

25. Perl, A. K. et al. Reduced expression of neural cell adhesion molecule induces metastatic dissemination of pancreatic beta tumor cells. Nature medicine 5, 286291 (1999).

26. Dahl, U., Sjodin, A. \& Semb, H. Cadherins regulate aggregation of pancreatic betacells in vivo. Development (Cambridge, England) 122, 2895-2902 (1996).

27. Kondo, J. et al. Retaining cell-cell contact enables preparation and culture of spheroids composed of pure primary cancer cells from colorectal cancer.
Proceedings of the National Academy of Sciences of the United States of America 108, 6235-6240 (2011).

28. Christofori, G., Naik, P. \& Hanahan, D. A second signal supplied by insulin-like growth factor II in oncogene-induced tumorigenesis. Nature 369, 414-418 (1994).

29. Tal, M. et al. Glucose transporter isotypes switch in T-antigen-transformed pancreatic beta cells growing in culture and in mice. Molecular and cellular biology 12, 422-432 (1992)

30. Maeda, M., Johnson, K. R. \& Wheelock, M. J. Cadherin switching: essential for behavioral but not morphological changes during an epithelium-to-mesenchyme transition. Journal of cell science 118, 873-887 (2005).

31. Sobhanifar, S., Aquino-Parsons, C., Stanbridge, E. J. \& Olive, P. Reduced expression of hypoxia-inducible factor-1alpha in perinecrotic regions of solid tumors. Cancer Res 65, 7259-7266 (2005).

32. Fukuda, R. et al. Insulin-like growth factor 1 induces hypoxia-inducible factor 1 mediated vascular endothelial growth factor expression, which is dependent on MAP kinase and phosphatidylinositol 3-kinase signaling in colon cancer cells. The Journal of biological chemistry 277, 38205-38211 (2002).

33. Soejima, A. et al. Mitochondrial DNA is required for regulation of glucosestimulated insulin secretion in a mouse pancreatic beta cell line, MIN6. The Journal of biological chemistry 271, 26194-26199 (1996).

34. Krishnamachary, B. et al. Hypoxia-inducible factor-1-dependent repression of Ecadherin in von Hippel-Lindau tumor suppressor-null renal cell carcinoma mediated by TCF3, ZFHX1A, and ZFHX1B. Cancer research 66, 2725-2731 (2006).

35. Yang, M. H. et al. Direct regulation of TWIST by HIF-1alpha promotes metastasis. Nature cell biology 10, 295-305 (2008).

36. Schietke, R. et al. The lysyl oxidases LOX and LOXL2 are necessary and sufficient to repress E-cadherin in hypoxia: insights into cellular transformation processes mediated by HIF-1. The Journal of biological chemistry 285, 6658-6669 (2010).

37. Wheelock, M. J., Shintani, Y., Maeda, M., Fukumoto, Y. \& Johnson, K. R. Cadherin switching. Journal of cell science 121, 727-735 (2008).

38. Couvelard, A. et al. Microvascular density and hypoxia-inducible factor pathway in pancreatic endocrine tumours: negative correlation of microvascular density and VEGF expression with tumour progression. British journal of cancer 92, 94$101(2005)$.

\section{Acknowledgements}

We thank Yoriko Mawatari, Momoko Shiozaki, and Noriko Kanto for technical assistance. We thank Oriol Casanovas, Allen Elizabeth Anne, and Hiroko Endo for critical reading of the manuscript. This work was supported by KAKENHI 21590459, and by the Naito foundation.

\section{Author contributions}

Masahiro Inoue (MI), Yasuko Nishizawa, and Shuhei Tomita (ST) designed the study. MI and Takaaki Takeda (TT) wrote the main manuscript text. TT, ST, Hiroaki Okuyama, YN and MI performed the experiments and prepared the figures. All authors reviewed the manuscript.

\section{Additional information}

Supplementary information accompanies this paper at http://www.nature.com/ scientificreports

Competing financial interests: The authors declare no competing financial interests. License: This work is licensed under a Creative Commons Attribution-NonCommercial-ShareAlike 3.0 Unported License. To view a copy of this license, visit http://creativecommons.org/licenses/by-nc-sa/3.0/

How to cite this article: Takeda, T., Okuyama, H., Nishizawa, Y., Tomita, S. \& Inoue, M. Hypoxia inducible factor- $1 \alpha$ is necessary for invasive phenotype in Vegf-deleted islet cell tumors. Sci. Rep. 2, 494; DOI:10.1038/srep00494 (2012). 